\title{
Autonomic cardiovascular response to acute hypoxia and passive head-up tilting in humans
}

\author{
S. J. Brown · A. Raman • M. J. Barnes • \\ T. Mündel
}

Received: 19 July 2012/Accepted: 29 January 2013/Published online: 12 February 2013

(c) Springer-Verlag Berlin Heidelberg 2013

\begin{abstract}
Acute hypoxia may alter autonomic cardiovascular reflexes during orthostasis. Heart rate variability (HRV), arterial blood pressure (MAP), and respiratory sinus arrhythmia (RSA) were recorded during supine (SUP) and passive head up tilt (HUT) in eight healthy humans, spontaneously breathing either room air or $10 \%$ $\mathrm{O}_{2}$ in $\mathrm{N}_{2}$. In the time domain, heart rate increased and variability decreased with HUT in both trials, with no difference between trials. In the frequency domain, normalized low frequency HRV increased, and normalized high frequency HRV decreased with HUT in both trials, with no difference between trials. MAP was 74.9 (8.6) and 77.5 (11.7) $\mathrm{mmHg}$ when SUP in the room air and hypoxia trials, respectively. A significant increase in MAP occurred with HUT in the room air trial but not in the hypoxia trial. In both trials, end tidal $\mathrm{CO}_{2}$ decreased with HUT, with no difference between trials. In the room air trial, end tidal $\mathrm{O}_{2}$ increased with HUT, whereas during the hypoxia trial, end tidal $\mathrm{O}_{2}$ decreased with HUT. The distribution of heart beats relative to the phase of ventilation $\left(\% \mathrm{HB}_{\mathrm{IN}}\right.$ and $\% \mathrm{HB}_{\text {OUT }}$ ) was similar in both trials: the $\% \mathrm{HB}_{\mathrm{IN}}$ was 43.5 (3.3) $\%$ and $\% \mathrm{HB}_{\text {OUT }}$ was 56.5 (4.2) $\%$ breathing room air when SUP, and 45.5 (3.0) and 54.5 (3.2) when hypoxic and SUP. For both trials, this distribution did not change with HUT. As both HRV and RSA showed similar responses to
\end{abstract}

Communicated by Massimo Pagani.

\section{S. J. Brown ( $\square)$}

School of Health Sciences, University of Ballarat,

University Drive, Mount Helen, Ballarat, VIC 3350, Australia

e-mail: bahrainstephen@gmail.com

A. Raman · M. J. Barnes · T. Mündel

School of Exercise and Sport Sciences, Massey University,

Palmerston North, New Zealand
HUT when spontaneously breathing either room air or $10 \% \mathrm{O}_{2}$ in $\mathrm{N}_{2}$, we suggest that autonomic cardiovascular reflexes are preserved during acute hypoxia.

Keywords Autonomic nervous system .

Spectral analysis - Cardiac autonomic control

\section{Introduction}

Alternating periods of increasing and decreasing heart rate in phase with breathing [respiratory sinus arrhythmia (RSA)] may improve the efficiency of gas exchange in the lungs (Hayano et al. 1996; Yasuma and Hayano 2004; Grossman and Taylor 2007). Increasing blood flow through the lung during inhalation by the preferential distribution of heart beats during inhalation may reduce intrapulmonary shunt, however, this phasic variation in heart rate is dependent on both body position (Brown et al. 2009a, b; Cooke et al. 1999) and pH (Brown and Howden 2008; Brown et al. 2007). Also, RSA has been shown to increase with hypercapnia but not hypoxemia (Tzeng et al. 2007), in part due to the stimulation of ventilation by an elevated $\mathrm{PaCO}_{2}$. The power of the high frequency (HF) component of heart rate variability (HRV) has been used as a measure of both cardiac vagal tone and RSA since normal resting respiratory frequency is within the HF range $(0.15-0.4 \mathrm{~Hz})$. Hypoxia may decrease both low frequency (LF) and HF power (Bernardi et al. 1998; Cornolo et al. 2004; Perini et al. 1996; Ponchia et al. 1994; Saito et al. 2005; Sevre et al. 2001); however, hypoxia may decrease HF power yet increase LF power (Hughson et al. 1994), or have no effect on HF power while increasing LF power (Iwasaki et al. 2006). These equivocal findings emphasize the complex interaction between both inspired $\mathrm{PO}_{2}$ and 
$\mathrm{PCO}_{2}$, and autonomic nervous system function (Hainsworth et al. 2007; Somers et al. 1991; Kamiya et al. 2005). For example, acute hypoxia may increase both heart rate and blood pressure, although paradoxically, hypoxemia may act on vascular smooth muscle in the systemic circulation causing vasodilation and hypotension. Also, recurrent hypoxic episodes may cause a sustained elevation of mean arterial pressure, although this hypertension appears to be independent of any accompanying hypercapnia. From a clinical perspective, chronic intermittent hypoxia during recurrent apnea syndromes may cause hypertension, increased sympathetic nerve activity, cardiac arrhythmias and myocardial infarction (Prabhakar et al. 2005)

Head-up tilt (HUT) represents a physiological challenge requiring coordinated cardiovascular responses including peripheral vasoconstriction and increasing heart rateprolonged passive HUT may induce vasovagal syncope. HUT also affects HRV, whereby HF power has consistently been shown to decrease in healthy humans (Cooke et al. 1999; Kamiya et al. 2005; Kochiadakis et al. 1998; Piccirillo et al. 1995). However, directional changes in LF power with HUT appear equivocal, where no changes (Cooke et al. 1999; Kamiya et al. 2005) or increases (Kochiadakis et al. 1998; Piccirillo et al. 1995) have been reported. Impaired cognitive function, spatial disorientation, and syncope, are all characteristics of both acute hypoxia and passive HUT. Indeed, an environment in which a reduced inspired $\mathrm{PO}_{2}$ occurs with a gravitationalinduced redistribution of blood volume will challenge the human body's ability to maintain homeostasis. However, there is currently a paucity of data on the combined effects of acute hypoxia and HUT on both HRV and RSA. Therefore, in the current study, we aimed to quantify these cardiovascular responses in healthy humans to provide further insight into the underlying mechanisms.

\section{Methods}

With informed consent, eight healthy adult non-smokers (age range 21-44 years, 4 males, 4 females), with no known cardiovascular or respiratory abnormalities were studied at rest. The female subjects were taking a progesterone-based contraceptive pill and were at least 1 week pre-menstrual. Subjects were at least $4 \mathrm{~h}$ post-prandial, and refrained from caffeine containing drinks in the preceding $4 \mathrm{~h}$. All procedures were approved by local Human Ethics Committee.

In a randomized order, subjects were required to breathe either room air (RA: $21 \% \mathrm{O}_{2}, 79 \% \mathrm{~N}_{2}$ ) or normobaric hypoxic air (HYPOXIA: $10 \% \mathrm{O}_{2}, 90 \% \mathrm{~N}_{2}$ ), for a total duration of $12 \mathrm{~min}$, at standard ambient room temperature and atmospheric pressure, i.e., $\sim 20{ }^{\circ} \mathrm{C}$ and $760 \mathrm{mmHg}$. Each 12 min epoch was sub-divided into two 6 min periods. During the first period subjects rested supine (SUP); this was followed by a period of $6 \mathrm{~min}$ passive HUT at $85^{\circ}$. Moving from the SUP to the HUT position took $<2 \mathrm{~s}$. An allocated washout period between gas exposures was approximately $5 \mathrm{~min}$-during this time subjects could remove the mouthpiece and breathe room air.

During exposures subjects were asked to breathe through a mouthpiece with nasal occlusion. The mouthpiece was connected to a pneumotach (MLT1000L Respiratory Flow Head, AD Instruments, Australia) and a low resistance demand valve connected to a 180L Douglas bag filled with either RA, or HYPOXIA. The mouthpiece and pneumotach added approximately $100 \mathrm{ml}$ ventilatory dead space to each subject. The fractional content of $\mathrm{O}_{2}$ and $\mathrm{CO}_{2}$ in the dried air (MLA6024 AD Instruments, Australia) flowing through the pneumotach was continuously measured (response time approximately $100 \mathrm{~ms}$ ) throughout inhalation and exhalation using a gas analyzer (ML206 AD Instruments, Australia). The $\mathrm{O}_{2}$ transducer used absorption spectroscopy at $\lambda 760 \mathrm{~nm}$ and the $\mathrm{CO}_{2}$ transducer used an infrared sensor. The analyzer was calibrated with samples of known $\mathrm{O}_{2}$ and $\mathrm{CO}_{2}$ gas mixtures prior to testing each subject. The length of the sampling lines from the pneumotach to analyzer were minimized to reduce the signal offset - in subsequent data analyses the chart recorder $\mathrm{O}_{2}$ and $\mathrm{CO}_{2}$ data streams were time shifted such that the initial changes from typical room air coincided with the start of exhalation, as defined by a continuous positive deflection in ventilatory flow. Ventilatory flow was integrated to determine volume, and this signal was calibrated using a $3-\mathrm{L}$ calibration syringe (Hans Rudolph, USA). Before each test, the pneumotach signal was reset to zero when disconnected from the subject. The mixed expired fractional content of $\mathrm{O}_{2}$ and $\mathrm{CO}_{2}$ and the expired end-tidal fractional content of $\mathrm{O}_{2}$ and $\mathrm{CO}_{2}$ were recorded.

An electrocardiogram (ECG limb lead 2, band-pass filtered between 10 and $200 \mathrm{~Hz}$, sampling frequency of $1 \mathrm{kHz}$ ) was recorded from each subject and collected using a multi-channel analog-to-digital data acquisition system with appropriate software (PowerLab 4/25T and Chart v5.4, AD Instruments, Australia). The ECG was recorded continuously, and was used for HRV analysis using commercially available software (HRV Module for Chart 5, AD Instruments, Australia). Heart rate was calculated by expressing $\mathrm{R}-\mathrm{R}$ intervals as beats per minute (beats $\left.\min ^{-1}\right)$. $\mathrm{R}-\mathrm{R}$ period data were re-sampled to generate a waveform with uniform time interval. Data were analyzed in the time domain using the mean $\mathrm{R}-\mathrm{R}$ interval, and the standard deviation of the normal mean $\mathrm{R}-\mathrm{R}$ interval, and in the frequency domain using a Fourier analysis and a Welch 
averaged periodogram method, and banded as very low frequency (VLF: $0-0.04 \mathrm{~Hz}), \mathrm{LF}(0.04-0.15 \mathrm{~Hz})$, and HF $(0.15-0.4 \mathrm{~Hz})$. Total power for each spectrum was defined as the area under the spectrum from 0 to $0.5 \mathrm{~Hz}$, and normalized units (\%) for the LF and HF components (which take into account any changes in total spectrum power) were calculated.

$\mathrm{R}$ waves were identified as occurring during the inhalatory phase $\left(\% \mathrm{HB}_{\mathrm{IN}}\right)$ or exhalatory phase $\left(\% \mathrm{HB}_{\mathrm{OUT}}\right)$ of ventilation using a peak detection and data logging software routine. These data were used to calculate the relative distribution of heart beats throughout the ventilatory cycle.

Blood pressure was recorded continuously with a noninvasive arterial monitor (Finometer, Ohmeda, UK), and the signal transferred electronically to the chart recorder. The recording was taken from a warmed finger placed at heart level as per manufacturer's instructions, and mean arterial pressure was determined as $1 / 3$ systolic $+2 / 3$ diastolic pressure.

Each variable of interest was analyzed using analysis of variance (significance level at 0.05 ) following confirmation of distribution normality, and if appropriate, with a post hoc paired sample Student's $t$ test to determine where differences occurred (minimum level of significance $P<0.03)$. Values reported are the mean $(\mathrm{SD})$.

\section{Results}

All subjects completed both trials, and no syncope episodes were recorded. Heart rate increased and variability decreased with HUT in both ROOM AIR and HYPOXIA trials (Table 1), with no difference between trials. In the time domain, mean $\mathrm{R}-\mathrm{R}$ decreased by approximately 30 and $25 \%$ in the ROOM AIR and HYPOXIA trials, respectively, and SD R-R decreased by approximately $30 \%$ in both trials. In the frequency domain, LF (\%) increased and HF (\%) decreased with HUT in both trials.

Changes in the low and HF components of HRV have been plotted against the tilt-induced change $(\Delta)$ in heart rate in Fig. 1. This figure describes the relationships between the tilt-induced change $(\Delta)$ in normalized frequency components of HRV and the tilt-induced change in heart rate. The upper part of the figure shows $\Delta \mathrm{LF}$ versus $\Delta \mathrm{HR}$ - these data indicate that for a larger $\Delta$ in heart rate with tilt (further to the right on the $x$ axis), the greater the $\Delta$ in LF (higher up the $y$ axis). However, the relationships are only weak, and the response to tilt for both the room air and the hypoxic conditions are not different. The lower part of the figure shows $\Delta \mathrm{HF}$ versus $\Delta \mathrm{HR}$ - these data show that the greater the increase in heart rate with HUT, the greater the decrease in HF (shift to lower values on the $y$ axis). Again, the relationships are only weak and appear to be very similar for both room air and hypoxic conditions.

As shown in Fig. 2, mean arterial blood pressure was 74.9 (8.6) and 77.5 (11.7) $\mathrm{mmHg}$ when SUP in the ROOM AIR and HYPOXIA trials, respectively. A significant increase $(P<0.03)$ in blood pressure occurred with HUT in the ROOM AIR trial but not in the HYPOXIA trial. Values for end tidal $\mathrm{CO}_{2}$ and $\mathrm{O}_{2}$ are also shown in Fig. 2. In both trials, end tidal $\mathrm{CO}_{2}$ decreased with HUT, with no difference between trials. In the ROOM AIR trial, end tidal $\mathrm{O}_{2}$ increased from 13.7 (1.2) to $14.4(0.8) \%$ with HUT $(P<0.03)$, whereas during the HYPOXIA trial, end tidal $\mathrm{O}_{2}$ decreased from $5.3(0.4)$ to $4.8(0.4) \%$ with HUT $(P<0.03)$.

The distribution of heart beats relative to the phase of ventilation $\left(\% \mathrm{HB}_{\mathrm{IN}}\right.$ and $\left.\% \mathrm{HB}_{\mathrm{OUT}}\right)$ was not different with HYPOXIA compared to ROOM AIR. The $\% \mathrm{HB}_{\text {IN }}$ was 43.5 (3.3) $\%$ and $\% \mathrm{HB}_{\text {OUT }}$ was 56.5 (4.2) \% breathing ROOM AIR when SUP, and the $\% \mathrm{HB}_{\text {IN }}$ was 45.5 (3.0) and $\% \mathrm{HB}_{\text {OUT }}$ was 54.5 (3.2) breathing HYPOXIA when SUP. Also, for both trials, there was no change in the distribution of heart beats relative to phases of ventilation with HUT.

\section{Discussion}

This study quantified autonomic cardiovascular responses to the combined stressors of acute hypoxia and passive head-up tilt in healthy humans during spontaneous breathing. The $\% \mathrm{HB}_{\text {IN }}$ and $\% \mathrm{HB}_{\text {OUT }}$ are novel measures to describe the distribution of heart beats relative to the phase of ventilation-this study is unique in that this method has been applied to the combined stressors of acute hypoxia and HUT. This study reported that the distribution of heart beats throughout phases of ventilation was unaffected by either hypoxia or head-up tilt. Also, this is the first study to report both MAP and $\Delta$ in HRV with combined hypoxia and HUT, and presents the novel finding of a tilt-induced decrease in the HF component of HRV yet no change in the distribution of heart beats relative to stages of ventilation, a finding consistent with RSA-vagal dissociation. A further unique finding of this study is that cardiac autonomic reflexes induced by orthostatic challenge appear to be preserved during acute hypoxia-albeit in healthy subjects.

The increased heart rate with reduced time domain variability during passive HUT is well documented (Cooke et al. 1999), as are the directional changes in frequency components of HRV (Kamiya et al. 2005). Hypoxia during HUT appeared to have no effect on these responses. Resting heart rate when supine was elevated during hypoxia, with no change in MAP - this is consistent with the homeostatic requirements imposed by hypoxia-induced peripheral vasodilatation. The elevation of MAP with HUT 
Table 1 Heart rate variability measures in both time and frequency domains during spontaneous breathing of atmospheric air (ROOM AIR) or normobaric hypoxia $\left(10 \% \mathrm{O}_{2}\right.$ in $\mathrm{N}_{2}$ ), when either resting supine (SUP) or during passive head-up whole body tilting to $80^{\circ}$ (HUT)

\begin{tabular}{|c|c|c|c|c|c|c|c|}
\hline & Mean R-R (ms) & SD R-R (ms) & Mean HR (beats $\min ^{-1}$ ) & $\mathrm{LF}\left(\mathrm{ms}^{2}\right)$ & $\mathrm{HF}\left(\mathrm{ms}^{2}\right)$ & $\mathrm{LF}(\%)$ & $\mathrm{HF}(\%)$ \\
\hline \multicolumn{8}{|l|}{ ROOM AIR } \\
\hline SUP & $1,073(178)$ & $96(42)$ & $63(17)$ & $3,345(3,599)$ & $6,130(5,083)$ & $36.3(23.6)$ & $61.0(22.6)$ \\
\hline HUT & $754(125)$ & $64(17)$ & $82(14)$ & $1,766(980)$ & $985(658)$ & $64.2(11.7)$ & $33.5(10.7)$ \\
\hline$\Delta$ (HUT-SUP) & & & $18.36(9.37)$ & & & $27.86(21.87)$ & $-27.56(20.44)$ \\
\hline \multicolumn{8}{|l|}{ HYPOXIA } \\
\hline SUP & $850(105)$ & $122(50)$ & $70(10)$ & $2,895(4,140)$ & $2,364(1,940)$ & $45.5(26.3)$ & $49.7(24.2)$ \\
\hline HUT & $639(60)$ & $87(58)$ & $95(9)$ & $1,653(1,139)$ & 706 (917) & $71.2(14.7)$ & $25.7(11.9)$ \\
\hline$\Delta$ (HUT-SUP) & & & $24.28(6.98)$ & & & $25.65(20.56)$ & $-24.02(19.36)$ \\
\hline
\end{tabular}

Values are mean (SD) for eight subjects
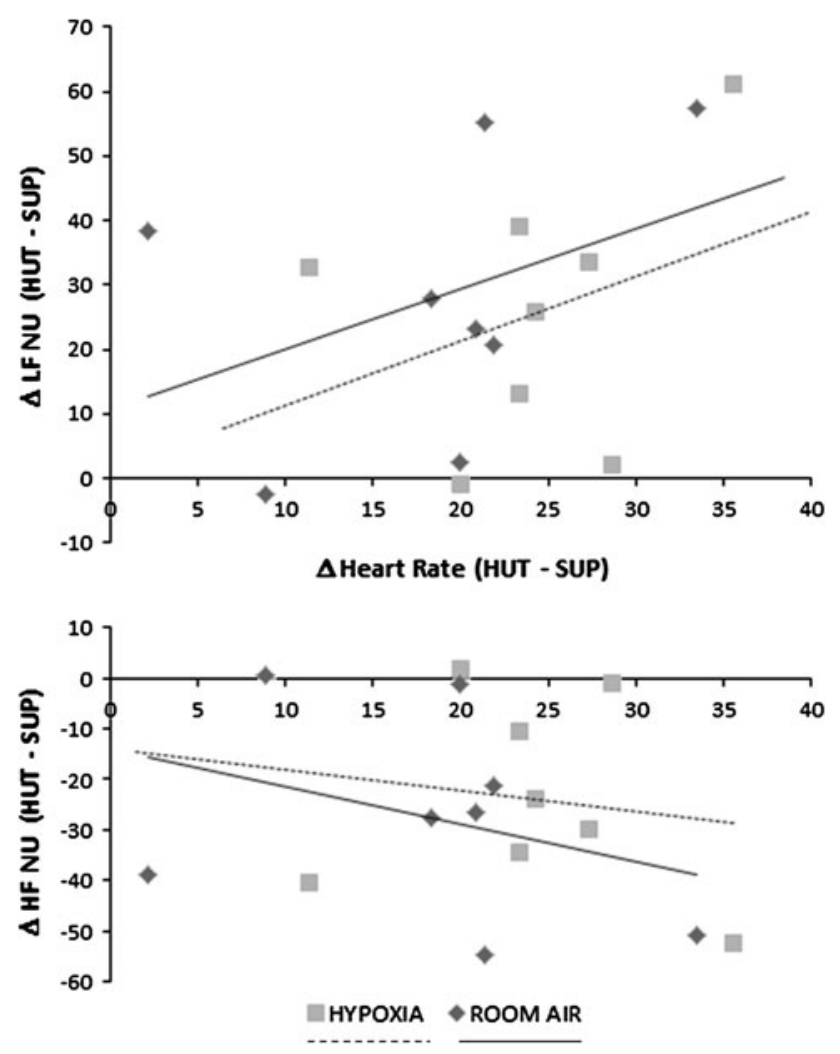

Fig. 1 Change $(\Delta)$ in low frequency $\left(\mathrm{LF}_{\mathrm{NU}}\right)$ and high frequency $\left(\mathrm{HF}_{\mathrm{NU}}\right)$ components of heart rate variability as a function of $\Delta$ in heart rate induced by passive head-up tilt (HUT), when spontaneously breathing either room air or $10 \% \mathrm{O}_{2}$ in $\mathrm{N}_{2}$ (Hypoxia)

in the room air trial was consistent with a tilt-induced peripheral vasoconstriction and increased heart ratereflexes concordant with the maintenance of cerebral perfusion (Rosner and Coley 1986; Toska and Walloe 2002). Hypoxia did not change MAP when SUP was compared to HUT, and it has previously been reported that mild systemic hypoxia had no effect on MAP, and did not affect baroreflex responses to neck suction or pressure (Eckberg et al. 1982).

In the current study, hypoxia was caused by spontaneous ventilation of $10 \% \mathrm{O}_{2}$ in $\mathrm{N}_{2}$, thus both pulmonary hypoxia and circulating hypoxemia are potential origins for changes in cardiac auto-regulation. In the hypoxia trial, MAP was not significantly increased with HUT, possibly indicating that a local hypoxemia in the peripheral circulation reduced or abolished any tilt-induced vasoconstriction. However, we can speculate that the tilt-induced increase in heart rate during the hypoxia trial was sufficient to maintain cerebral perfusion and prevent the onset of syncope. Acute hypoxia increases sympathetic activity and may increase both heart rate and blood pressure (Hainsworth et al. 2007)responses possibly attributable to elevated circulating catecholamine concentration (Mazzeo et al. 1998). Paradoxically, hypoxia also acts on vascular smooth muscle in the systemic circulation (Marshall 1994), potentially causing vasodilation and hypotension. In the current study, MAP did not increase with hypoxia despite an increase in heart rate. We suggest that the increased heart rate is mediated by the arterial baroreflex, whereby arterial pressure is maintained despite a possible reduction in total peripheral resistance initiated by hypoxia-induced peripheral vasodilation. $\mathrm{HF}_{\mathrm{NU}}$ was unchanged with hypoxia when compared to room air, and there was no evidence of any changes in the distribution of heart beats during either the inhalatory or exhalatory phases of ventilation with hypoxia - these findings suggest that hypoxia had no effect on either vagal tone (Hirsch and Bishop 1981) or RSA.

In the current study, MAP remained above $50 \mathrm{mmHg}$ in all subjects throughout SUP and HUT. Lower values of cerebral tissue oxygenation may be more common with a systolic blood pressure lower than $80 \mathrm{mmHg}$ during HUT (Suzuki et al. 2008), and it has been suggested that systolic pressures $<80 \mathrm{mmHg}$ and MAP $<60 \mathrm{mmHg}$ are the lower 
Fig. 2 Mean (SD) tilt-induced changes in mean arterial blood pressure (MAP), end tidal $\mathrm{CO}_{2}$, and end tidal $\mathrm{O}_{2}$, when spontaneously breathing either room air or $10 \% \mathrm{O}_{2}$ in $\mathrm{N}_{2}$ (Hypoxia). Values are the mean for eight subjects when supine (SUP) and when in a head up tilt position (HUT)
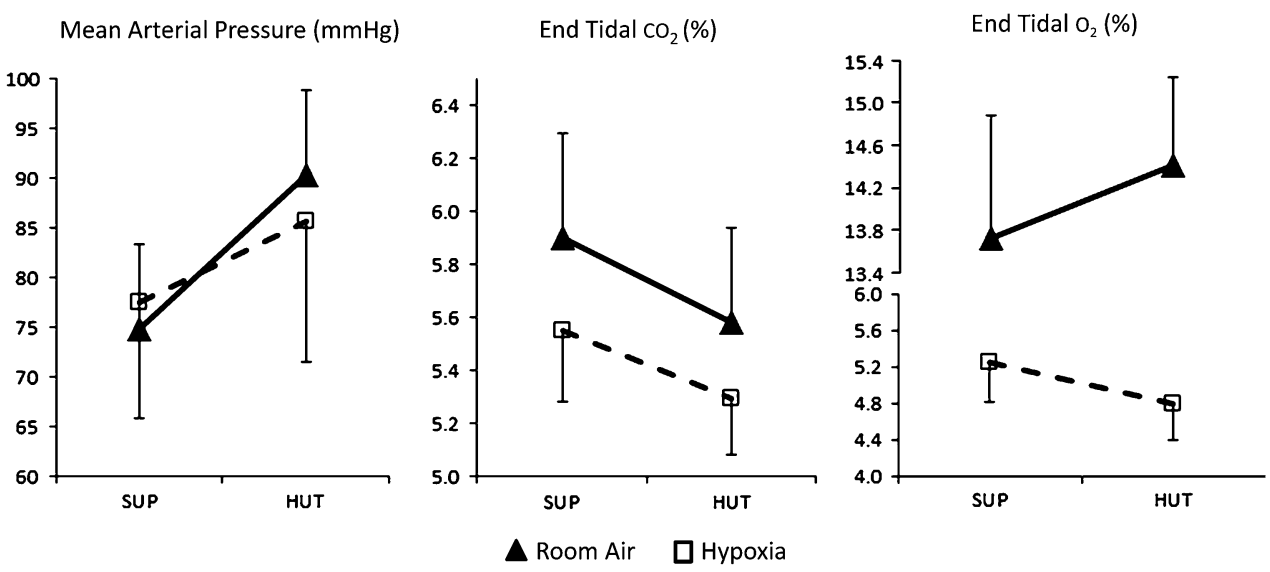

limits for cerebrovascular autoregulation (Novak et al. 1998). However, others (Thomas et al. 2010) have shown independence of both systolic blood pressure and MAP on cerebral oxygenation during cerebral hypo-perfusion.

In the current study, normobaric hypoxia using $10 \% \mathrm{O}_{2}$ in $\mathrm{N}_{2}$ is equivalent to an inspired $\mathrm{PO}_{2}$ of approximately $70 \mathrm{mmHg}$ - this acute hypoxia will potentially cause pulmonary vasoconstriction and pulmonary hypertension, and severely limit the capacity for physical work. Without acclimatization, such a level of hypoxia is poorly tolerated and may lead to adverse physiological consequences if prolonged. All our subjects tolerated the hypoxia for the required period, but signs of pre-syncope were evident in some (two) subjects during HUT when hypoxic. Thus, although our results suggest the preservation of autonomic integrity during combined orthostatic challenge and hypoxia, similarly reported by others (Rickards and Newman 2002; Henriksen and Rowell 1986), prolonged exposure to acute hypoxia (without acclimatization) and HUT may decrease orthostatic tolerance. Although the work carried out in the current study was on healthy subjects, brief periods of hypoxia may be experienced during sleep apnea (SA) and chronic obstructive pulmonary disease (COPD) - thus, the data presented here may have relevance in the study of these conditions. The data presented in this study support the notion that typical cardiovascular reflexes in healthy subjects are preserved in acute hypoxia, however, we speculate that clinical populations (SA and COPD) may not have this preservation, and may experience altered autonomic regulation. No data are available to support this suggestion, but we suggest that initially studying the healthy may provide an ideal starting point to perform these studies on patients.

Hypoxia induced by inhalation of $10 \% \mathrm{O}_{2}$ in $\mathrm{N}_{2}$ has some similarities with breath-hold diving-a procedure shown to influence both HRV and RSA (Lemaitre et al. 2008). However, in the current study, subjects maintained pulmonary ventilation during the acute hypoxia exposure, and thus the respiratory effect on both HRV components and RSA were potentially modified by changes to ventilatory patterns - this is different to the hypoxia induced by breath holding. We also imposed an orthostatic challenge with passive, whole body, head-up tilt. During submerged diving procedures (both free diving and SCUBA), ambient hydrostatic pressure induces a shift in blood volume which can increase central venous pressure and venous return which is in contrast to the typical cardiovascular responses to orthostatic challenges.

A decrease in end tidal $\mathrm{PCO}_{2}$ with HUT reflects the distribution of pulmonary perfusion relative to ventilation. An upright lung has under-perfused but ventilated alveoli in the proximal regions of the lung, adding physiological dead space, therefore end tidal $\mathrm{PCO}_{2}$ is reduced due to mixing with ambient air. A surprising result in the present study was the decrease in end tidal $\% \mathrm{O}_{2}$ with HUT in the hypoxia trial. Mixing of end tidal exhaled gas with ambient inhaled air containing $10 \% \mathrm{O}_{2}$ should increase end tidal $\% \mathrm{O}_{2}$ with $\mathrm{HUT}$, as shown in the room air trial. Although speculative, we suggest that hypoxia-induced pulmonary vasoconstriction may reduce physiological dead space in the upright lung-a mechanism which potentially improves ventilatory efficiency when hypoxic.

In summary, these data suggest that cardiovascular reflexes and cardiac autonomic regulation are preserved during acute hypoxia. The vagal withdrawal associated with moving from a supine to a passive head-up position appeared unaffected by acute hypoxia, however, the appearance of pre-syncope signs in some subjects during HUT when hypoxic requires further investigation.

\section{References}

Bernardi L, Passino C, Spadacini G et al (1998) Cardiovascular autonomic modulation and activity of carotid baroreceptors at altitude. Clin Sci 95:565-573 
Brown SJ, Howden R (2008) The effects of a respiratory acidosis on human heart rate variability. Adv Exp Med Biol 605:361-365

Brown SJ, Munel T, Brown JA (2007) Cardiac vagal control and respiratory sinus arrhythmia during hypercapnia in humans. J Physiol Sci 57:337-342

Brown SJ, Bryant M, Mündel T, Stannard SR (2009a) Human ventilatory efficiency and respiratory sinus arrhythmia during head-up tilt. J Physiol Pharm 59:771-780

Brown SJ, Mündel T, Barnes M, Brown JA (2009b) Tilt-induced changes in human heart rate variability with and without a respiratory acidosis. J Physiol Sci 59:31-36

Cooke WH, Hoag JB, Crossman AA, Kuusela TA, Tahvanainen KUO, Eckberg DL (1999) Human responses to upright tilt: a window on central autonomic integration. J Physiol 517:617-628

Cornolo J, Mollard P, Brugniaux JV et al (2004) Autonomic control of the cardiovascular system during acclimatization to high altitude: effects of sildenafil. J Appl Physiol 97:935-940

Eckberg DL, Barstow H, Scruby AE (1982) Modulation of human sinus node function by systemic hypoxia. J Appl Physiol 52:570-577

Grossman P, Taylor E (2007) Toward understanding respiratory sinus arrhythmia: relations to cardiac vagal tone, evolution and biobehavioural functions. Biol Psych 74:263-285

Hainsworth R, Drinkhill MJ, Rivera-Chira M (2007) The autonomic nervous system at high altitude. Clin Auton Res 17:13-19

Hayano J, Yasuma F, Okada A, Mukai S, Fujinami T (1996) Respiratory sinus arrhythmia-phenomenon improving pulmonary gas exchange and circulatory efficiency. Circulation 94:842-847

Henriksen O, Rowell LB (1986) Lack of effect of moderate hypoxaemia on human postural reflexes to skeletal muscle. Acta Physiol Scand 127:171-175

Hirsch JA, Bishop B (1981) Respiratory sinus arrhythmia in humans: how breathing pattern modulates heart rate. Am J Physiol Heart Circ Physiol 241:H620-H629

Hughson RL, Yamamoto Y, McCullough RE et al (1994) Sympathetic and parasympathetic indicators of heart rate control at altitude studied by spectral analysis. J Appl Physiol 77:2537-2542

Iwasaki K, Ogawa Y, Aoki K et al (2006) Cardiovascular regulation response to hypoxia during stepwise decreases from 21 to $15 \%$ inhaled oxygen. Aviat Space Environ Med 77:1015-1019

Kamiya A, Hayano J, Kawada $\mathrm{T}$ et al (2005) Low-frequency oscillation of sympathetic nerve activity decreases during development of tilt-induced syncope preceding sympathetic withdrawal and bradycardia. Am J Physiol Heart Circ Physiol 289:H1758-H1769

Kochiadakis GE, Kanoupakis EM, Igoumenidis NE, Marketou ME, Solomou MC, Vardas PE (1998) Spectral analysis of heart rate variability during tilt-table testing in patients with vasovagal syncope. Int J Cardiol 64:185-194

Lemaitre F, Buchheit M, Joulia F, Fontanari P, Tourny-Chollet C (2008) Static apnea effect on heart rate and its variability in elite breath-hold divers. Aviat Space Environ Med 79:99-104
Marshall JM (1994) Peripheral chemoreceptors and cardiovascular regulation. Physiol Rev 74:543-594

Mazzeo RS, Child A, Butterfield GE et al (1998) Catecholamine response during 12 days of high-altitude exposure $(4,300 \mathrm{~m})$ in women. J Appl Physiol 84:1151-1157

Novak V, Novak P, Spies JM, Low PA (1998) Autoregulation of cerebral blood flow in orthostatic hypotension. Stroke 29:104-111

Perini R, Milesi S, Biancardi L, Veicsteinas A (1996) Effects of high altitude acclimatization on heart rate variability in resting humans. Eur J Appl Physiol Occup Physiol 73:521-528

Piccirillo G, Fimognari F, Viola E, Marigliano V (1995) Age-adjusted normal confidence intervals for heart rate variability in healthy subjects during head-up tilt. Int J Cardiol 50:117-124

Ponchia A, Noventa D, Bertaglia M et al (1994) Cardiovascular neural regulation during and after prolonged high altitude exposure. Eur Heart J 15:1463-1469

Prabhakar NR, Peng Y, Jacono F, Kumar G, Dick T (2005) Cardiovascular alterations by chronic intermittent hypoxia: importance of carotid body chemoreceptors. Clin Exp Pharm Physiol 32:447-449

Rickards CA, Newman DG (2002) The effect of low-level normobaric hypoxia on orthostatic responses. Aviat Space Environ Med 73:460-465

Rosner MJ, Coley IB (1986) Cerebral perfusion pressure, intracranial pressure, and head elevation. J Neurosurg 65:636-641

Saito S, Tanobe K, Yamada M, Nishihara F (2005) Relationship between arterial oxygen saturation and heart rate variability at high altitudes. Am J Emerg Med 23:8-12

Sevre K, Bendz B, Hankø E et al (2001) Reduced autonomic activity during stepwise exposure to high altitude. Acta Physiol Scand 173:409-417

Somers VK, Mark AL, Abboud FM (1991) Interaction of baroreceptor and chemoreceptor reflex control of sympathetic nerve activity in normal humans. J Clin Invest 87:1953-1957

Suzuki K, Asahina M, Suzuki A, Hattori T (2008) Cerebral oxygenation monitoring for detecting critical cerebral hypoperfusion in patients with multiple system atrophy during head-up tilt test. Inter Med 47:1681-1687

Thomas KN, Galvin SD, Williams MJA, Willie CK, Ainslie PN (2010) Identical pattern of cerebral hypoperfusion during different types of syncope. J Hum Hypertension 24:458-466

Toska K, Walloe L (2002) Dynamic time course of hemodynamic responses after passive head-up tilt and tilt back to supine position. J Appl Physiol 92:1671-1676

Tzeng YC, Larsen PD, Galletly DC (2007) Effects of hypercapnia and hypoxemia on respiratory sinus arrhythmia in conscious humans during spontaneous respiration. Am J Physiol Heart Circ Physiol 292:H2397-H2407

Yasuma F, Hayano J (2004) Respiratory sinus arrhythmia—why does the heart beat synchronize with respiratory rhythm? Chest 125:683-690 